Original Paper http://ajol.info/index.php/ijbes http://indexmedicus.afro.who.int

\title{
Dynamique de la biodiversité des parcs agroforestiers de Vipalogo en zone nord soudanienne du Burkina Faso
}

\author{
Georges YAMEOGO ${ }^{1}$, Hamado OUEDRAOGO ${ }^{2}$ et Barthélémy YELEMOU ${ }^{3 *}$ \\ ${ }^{I}$ Institut de l'Environnement et de Recherches Agricoles (INERA), Cellule Télédétection et d'Information \\ Géographique (CTIG) 01 BP 476 Ouagadougou Burkina Faso. \\ ${ }^{2}$ Ministère des Ressources animales et halieutiques, Ouagadougou, Burkina Faso. \\ ${ }^{3}$ Institut de l'Environnement et de Recherches Agricoles (INERA)-Saria, BP 10 Koudougou, Burkina Faso. \\ "Auteur correspondant ; E-mail: yelbart@hotmail.com
}

\section{RESUME}

Une dynamique positive d'évolution des parcs agroforestiers constitue un critère important de résilience des populations rurales. Notre étude a porté sur l'analyse de l'évolution des modes de gestion des ressources naturelles et la dynamique du potentiel ligneux 10 ans après la création des parcs à Vipalogo, à travers une enquête socio-économique et un inventaire des ressources ligneuses. L'enquête semi structurée auprès de 50 chefs de ménages, a porté sur les modes de gestion des ressources ligneuses. L'inventaire floristique a concerné 14 champs mis en culture depuis 2004. Nos résultats montrent que le potentiel ligneux des 14 champs est passé de 434 arbres en 2004 à 339 en 2014, soit une baisse de 21,89\% et une diminution de la diversité spécifique qui est passée de 37 espèces en 2004 à 33 en 2014. La superficie totale des quatorze (14) parcs a connu une extension. En effet, elle est passée de 29,91 ha 2004 à 41,10 ha en 2014. En outre, le parc agroforestier connait une régression de la régénération et des individus de classe moyenne [20-30 cm [et [30$40 \mathrm{~cm}$ [. Pour une gestion durable des ressources, il faut palier à la régression des parcs agroforestiers.

(C) 2019 International Formulae Group. All rights reserved.

Mots clés : Parcs agroforestiers, inventaire, densité, biodiversité, évolution.

\section{Biodiversity dynamic of Vipalogo agroforestry parklands in north soudanian zone of Burkina Faso}

\section{ABSTRACT}

A positive dynamics of agroforestry parklands constitutes an important criterion of rural populations' resilience. Our study focused on analysis of natural resources management options and woody species potential dynamics, 10 years after agroforestry parkland creation in Vipalogo, through a socio-economic survey and woody species inventory. Semi-structured survey of 50 household heads focused on woody species management options. The floristic inventory concerned 14 fields cultivated since 2004. Our results show that woody species potential of the 14 farms decreased from 434 trees in 2004 to 339 in 2014, a proportion of $21.89 \%$ and a decrease in species diversity which went from 37 species in 2004 to 33 in 2014. The total area of the fourteen (14) parks has expanded. Indeed, it went from 29.91 ha 2004 to 41.10 ha in 2014. In addition, the agroforestry parkland is experiencing regression of regeneration and middle-class individuals in diameter classes [20-30 cm [and [30-40 cm [. For a sustainable management, agroforestry parkland woody species regression has to be stopped.

Keywords : Agroforestry parklands, inventory, density, biodiversity, dynamics. 


\section{INTRODUCTION}

La dynamique de l'interaction entre l'Homme et son écosystème en zone tropicale a toujours été influencée dans le temps par l'explosion démographique, qui elle-même influence les pratiques culturales (Paré, 2013). L'accroissement de la population a eu un impact significatif sur le changement du paysage des parcs agroforestiers du Burkina Faso, et le terroir de Vipalogo n'échappe pas à cette situation. Situé non loin de Ouagadougou, la capitale du Burkina Faso, Vipalogo connait une forte explosion démographique et par conséquent une forte occupation de son milieu physique.

En effet, la pression foncière est de plus en plus forte de même que l'empreinte écologique dans le paysage (Yaméogo et al., 2005). Un inventaire floristique réalisé en 2005 à Vipalogo dans des jachères agricoles a montré une forte représentativité de Combrétacées dans la végétation ligneuse, preuve d'une forte anthropisation et un appauvrissement des sols (Yaméogo et al., 2005).

Les populations, conscientes de la précarisation de leurs conditions de vie adoptent des comportements en faveur de la durabilité des ressources naturelles.

En 2004 (et travaux publiés en 2005), Yaméogo et al. (2005) étaient témoins de la création de quatorze (14) nouvelles défriches en parcs agroforestiers à partir d'anciennes jachères. La structure, la composition et le fonctionnement de ces nouvelles défriches ont été analysées par ces auteurs. Il ressort que seulement $11,7 \%$ des espèces végétales initialement présentes dans les jachères étaient conservées dans les nouveaux parcs agroforestiers, le reste des arbres étaient coupés ou brulés. Toutefois, la densité des arbres restés dans ces quatorze (14) parcs agroforestiers nouvellement créés demeurait toujours supérieure à celle des arbres dans les vieux parcs agroforestiers qui étaient en exploitation dans le même paysage forestier. Ce qui signifie que les paysans de Vipalogo ont compris qu'il faut garder plus d'arbres dans les nouvelles défriches. Cette amélioration des perceptions paysannes dans la création et la gestion des nouveaux parcs agroforestiers a été possible avec le soutien d'un certain nombre de projets de développement mis en œuvre dans la zone à travers les appui-conseils et encadrements techniques (Yaméogo, 2008).

Cependant de nos jours, sous la pression des différentes charges de fonctionnement de la famille (besoins en bois énergie, en bois de construction, en espace agricole, en productivité), les populations, malgré la connaissance du bien-fondé de la préservation de l'environnement (Yaméogo, 2008), sont de plus en plus enclins à couper les arbres. Les raisons qui justifiaient le fait qu'un arbre était coupé ou épargné lors d'une nouvelle défriche sont difficilement défendables. Les populations cèdent-elles à la satisfaction des besoins plus urgents? En effet, la plupart des essences agroforestières qui étaient soigneusement sélectionnées pour être maintenues sont de plus en plus coupées ou brûlées (Naboho, 2007). Les stratégies paysannes de gestion des ressources ligneuses et non ligneuses issues des parcs agroforestiers (champs) sont en perpétuelle mutation et influencent souvent négativement la dynamique de la structure, la composition et le fonctionnement des parcs agroforestiers dans la région du plateau central (Boffa 2000 ; Guegant et al., 2003).

Au regard de ces problèmes de gestion des ressources agroforestières à Vipalogo, cette étude a été initiée et vise à situer l'état d'évolution des parcs du terroir après dix ans de gestion et de mieux comprendre la logique paysanne de gestion des ressources naturelles.

\section{MATERIEL ET METHODES}

\section{Localisation et caractérisation du site}

Le site de l'étude est Vipalogo, un village situé à $40 \mathrm{~km}$ au Sud-Ouest de Ouagadougou (capitale du Burkina Faso et chef-lieu de la province du Kadiogo). Il est compris entre la latitude $12^{\circ} 04^{\prime} 20^{\prime \prime}$ et $12^{\circ} 09^{\prime} 30^{\prime \prime}$ Nord et $1^{\circ} 42^{\prime} 50^{\prime \prime}$ et $1^{\circ} 49^{\prime} 00^{\prime \prime}$ Ouest (Figure 1).

Le terroir de Vipalogo fait partie du grand ensemble généralement appelé «Plateau Central » qui se caractérise par une forte concentration humaine de 76 habitants au $\mathrm{km}^{2}$, contre une moyenne nationale de 38 habitants au $\mathrm{km}^{2}$ (Ministère de l'Economie et des Finances, 2009). Vipalogo appartient au 
domaine phytogéographique Nord-Soudanien (Sambaré et al., 2010; Thiombiano et Kampmann 2010) caractérisé par des savanes arborées et arbustives à Vitellaria paradoxa Gaertn.f.. Lannea microcarpa Engl. \& K. Krause, Parkia biglobosa (Jacq.) R.Br. ex G.Don.,Faidherbia albida (Del.) Chev., Tamarindus indica L., Combretum micranthum G. Don, Combretum glutinosum Perr. ex DC., Guiera senegalensis J.F.Gmel., Acacia seyal Del. Le paysage est fortement marqué par l'emprise humaine, illustrée par la présence de savanes parcs et de jachères à karité (Vitellaria paradoxa) et à néré (Parkia biglobosa).

Le climat est caractérisé par une saison sèche de sept mois (de novembre à mai) et une saison pluvieuse de cinq mois (de juin à octobre). La pluviométrie annuelle est très variable et oscille entre $600 \mathrm{~mm}$ et 1000 $\mathrm{mm}$, avec une moyenne de $761 \mathrm{~mm}$ sur les trente dernières années (Yaméogo et al., 2005). Les températures moyennes mensuelles connaissent également une variation allant de $24{ }^{\circ} \mathrm{C}$ à $33^{\circ} \mathrm{C}$.

\section{Méthodologie}

Pour atteindre les objectifs de l'étude, une enquête sur la gestion du parc agroforestier de même qu'un inventaire ont été réalisés dans le terroir de Vipalogo.

\section{Méthodologie de l'enquête}

Un échantillon significatif de la population a été déterminé afin de récolter des données plus précises. L'unité statistique primaire de cette enquête est l'exploitation agricole. L'ensemble des exploitations agricoles du village constitue la population de départ.

En outre, l'enquête a ciblé prioritairement et de façon raisonnée des exploitants (petits et grands) détenteurs de parcs agroforestiers parmi lesquels figurent les propriétaires des parcs agroforestiers qui ont été créés en 2004 (Yaméogo et al., 2005). Au total 50 responsables d'exploitations familiales ont été interviewés. Les questionnaires s'articulaient autour de trois axes principaux qui sont, les caractéristiques des systèmes de production impliquant les parcs agroforestiers, l'exploitation des arbres du parc et les autres facteurs déterminants de la gestion des parcs agroforestiers (la pression démographique, les feux, le pâturage, etc.).

Des entretiens semi-structurés ont été réalisés auprès des personnes ressources, des autorités administratives, communales, religieuses et coutumières.

\section{Inventaire du parc agroforestier}

Les champs retenus pour l'inventaire sont les mêmes qui ont été inventoriés il y a 10 ans par Yaméogo et al. (2005) et l'inventaire de la végétation ligneuse a eu lieu après les récoltes (en février). La superficie actuelle de chaque champ a été relevée. Dans chaque champ, des placettes de $50 \mathrm{~m}^{2}$ espacées de $10 \mathrm{~m}$ le long d'un transect, ont été posé. Dans chaque placette, les différentes espèces ont été notées de même que le nombre d'individus. Sur chaque individu, les mesures ont porté sur le diamètre à hauteur de poitrine, la hauteur totale de l'arbre, et le diamètre du houppier.

\section{Analyse statistique}

Les données d'enquêtes ont été traitées dans le logiciel SPSS.18. En considérant les mêmes parcs agroforestiers créés depuis 2004, des tests T de Student ont été faits sur la base des paramètres de structure de la population ligneuse des parcs agroforestiers. Lesdits tests ont permis de comparer les structures des parcs agroforestiers entre 2004 et 2014 et apprécier ainsi la dynamique évolutive.

Pour apprécier l'ampleur de la différence, nous avons calculé la taille de l'effet à partir de l'Eta-carré:

$$
\text { Eta-carré }=\mathrm{t}^{2} /\left(\mathrm{t}^{2}+\mathrm{N}-1\right)=
$$

$(2,729)^{2} /\left((2,729)^{2}+14-1\right)=0,363$

Selon, Cohen (1988), si le résultat de l'Eta-carré est :

- autour de 0,01, l'effet de la variable explicative est de petite taille (small effect)

- autour de 0,06 l'effet de la variable explicative est de taille moyenne (moderate effect)

- autour de 0,14 et plus, l'effet de la variable explicative est de grande taille (large effect). 


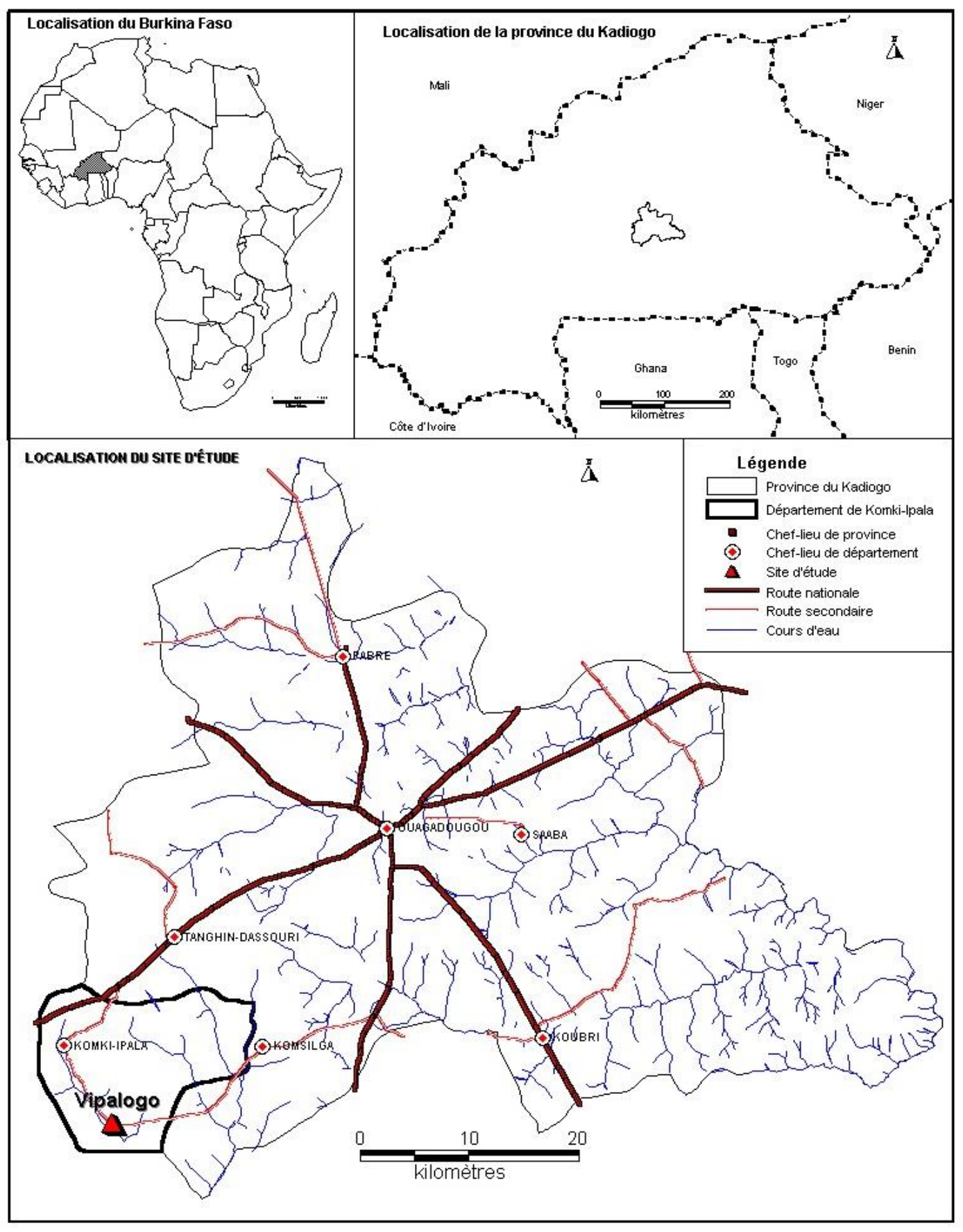

Figure 1 : Localisation du site d'étude.

\section{RESULTATS}

\section{Structure des exploitations.}

La taille moyenne des exploitations n'a pas sensiblement varié depuis dix ans. Elle est de huit (08) personnes par exploitation (Tableau 1). Toutefois, les écarts types montrent qu'il y a une grande dispersion entre les tailles des exploitations. Pour les populations enquêtées, les facteurs explicatifs de l'évolution de la structure des exploitations sont les naissances et les décès pour 7,5\%, les mariages représentent $27,5 \%$ et l'immigration, 
2,5\%. En outre, malgré la relative stabilité de la taille des exploitations agricoles, on assiste à une extension des superficies des parcs agroforestiers. La totalité des enquêtés $(100 \%)$, affirment avoir encore la possibilité d'étendre la superficie de leur parc agroforestier.

\section{Superficies exploitées, statut des exploitants et mode d'accès à la terre}

L'enquête a montré que les superficies exploitées par les populations de Vipalogo varient en moyenne entre 0,5 et 7,5 ha et les principales spéculations sont par ordre d'importance le sorgho, le petit mil, le maïs et l'arachide. Les exploitants pratiquent l'élevage de petits et gros ruminants, la volaille avec des races locales, faisant d'eux des agropasteurs. Le mode d'accès à la terre se fait prioritairement par héritage et rarement par prêt. De tous les enquêtés, seulement 30\% étaient des ayants droits fonciers, pour avoir directement hérité des parcs agroforestiers de leurs parents. Parmi ces 30\% des ayants droits, figuraient, deux veuves qui n'ont pas été expropriées de leurs lopins de terre après le décès de leur mari, ce qui est rare en terroir mossi où selon leurs coutumes et us, la femme ne peut pas hériter de la terre de son défunt mari. Soixante-dix (70\%) des enquêtés sont des propriétaires vivant et jouissant de tous les droits sur leurs parcs agroforestiers. Trentequatre pour cent (34\%) des enquêtés ont leurs parcs agroforestiers localisés à proximité des concessions, contre $50 \%$ des enquêtés qui ont leurs parcs agroforestiers en brousse. Seulement $16 \%$ des populations ont leurs champs situés au niveau des bas-fonds.

\section{Dynamique du potentiel ligneux du terroir de Vipalogo \\ Densité et abondance spécifiques}

La mise en culture des parcs agroforestiers de Vipalogo a entrainé une extension des superficies des champs suivis d'une diminution de la densité des arbres dans le parc qui passe de 14 pieds/ha en 2004 à 8,25 pieds/ha en 2014 , soit une réduction de $41 \%$.
On note une baisse sensible de l'abondance. En effet, l'inventaire des 14 parcs a concerné une superficie totale de 41,10 ha, permettant de dénombrer 339 individus, répartis en 33 espèces dont les plus abondantes sont Vitellaria paradoxa Gaern.f. (37,5\%), Lannea microcarpa Engl. \& Kraus (12,4\%), Parkia biglobosa (Jacq.) Benth., $(11,8 \%)$ et Azadirachta indica A. Juss $(11,2 \%)$ (Tableau 2).

Le Tableau 2 montre qu'en dix ans d'exploitation des parcs, $32 \%$ des espèces qui étaient présentes en 2004 ont disparus. Il s'agit entre autres de Acacia sieberiana, Sclerocarya birrea, Detarium microcarpa, Lannea acida, Acacia macrostachya, Albizia chevalieri.

Le suivi des 10 principales espèces dix ans après la création des parcs de Vipalogo, montre une diversité d'évolution suivant les espèces. En effet, à travers la Figure 2 nous remarquons que les espèces telles que Vitellaria paradoxa, Azadiracta indica et Parkia biglobosa ont régressés en nombre d'individus par contre Lannea microcarpa a progressée après dix années de culture.

\section{Dynamique de l'effectif des arbres dans 14 parcs agroforestiers entre 2004 et 2014}

Pour les quatorze (14) champs, l'effectif moyen des arbres est passé de $31,14 \pm 21,41$ arbres en 2004 à $21,64 \pm 10,58$ arbres après dix (10) ans de mise en culture. L'étude de corrélation effectuée entre les deux groupes de mesure auprès des 14 champs donne un coefficient de corrélation élevé ( $\mathrm{r}=$ $0,884)$, et la corrélation entre les deux groupes est très significative avec $p=0,000(p<0,05)$

La différence entre les deux moyennes est de 9,5 pieds. En outre il existe une différence significative entre l'effectif des arbres dans les champs avant et après dix (10) ans de mise en culture. L'état carré calculé est de 0,363. Cette analyse complémentaire confirme que la taille de l'effet associée à la dynamique végétative est très grande.

\section{Dynamique de la diversité spécifique dans 14 parcs agroforestiers entre 2004 et 2014 \\ Dans les quatorze (14) champs, le nombre moyen d'espèces est passé de $6,5 \pm 0,65$ en 2004 à $7,2 \pm 1,18$ après dix (10)}


ans de mise en culture. Et l'écart-type indique une faible dispersion entre les résultats avant et après les dix (10) ans de mise en culture (Tableau 3).

L'analyse statistique donne un coefficient de corrélation $r$ faible $(r=0,192)$. La corrélation entre les deux groupes n'est pas significative $(p=0,511)$. Cela conforte les résultats du coefficient de corrélation $r$.

\section{Dynamique de la structure des 14 parcs} agroforestiers de Vipalogo de 2004 à 2014

La structure des ligneux des parcs agroforestiers du terroir de Vipalogo en 2004, est marquée par une prédominance des individus de classe de diamètre compris entre [0- $10 \mathrm{~cm}$ [ et celle des ligneux de gros diamètre [40-et plus[ (Figure 3). Les individus de classe de diamètre moyen situé entre ces deux extrêmes demeurent moyennement représentés. L'allure de la courbe d'évolution des classes de diamètre en 2004 est incurvée. Dix ans après la mise en culture, le parc agroforestier connait une forte régression de la régénération des individus de classe moyenne [20- $30 \mathrm{~cm}[\mathrm{et}[30-40 \mathrm{~cm}[$.

Distribution des espèces par classe de diamètre dans les parcs agroforestiers de Vipalogo

La distribution des espèces du parc de Vipalogo, laisse voir une forte représentativité surtout pour trois espèces locales que sont Vitellaria paradoxa, Parkia biglobosa et Lannea microcarpa et une espèce exotique, Azadirachta indica (Figure 4). En outre, chez ces espèces bien présentes dans le parc, les individus de classe de diamètre variant de [40-et plus [sont les plus notables. Les principales espèces des parcs agroforestiers de Vipalogo sont de vieux sujets.

Tableau 1: Paramètres statistique de l'évolution de la taille des exploitations.

\begin{tabular}{ccc}
\hline & Taille actuelle de l'exploitation & Taille il y a 10 ans \\
\hline Moyenne & 8,12 & 8,83 \\
Médiane & 7,00 & 8,00 \\
déviation standard & 5,021 & 4,87 \\
Minimum & 1 & 2 \\
Maximum & 20 & 23 \\
\hline
\end{tabular}

Tableau 2 : Evolution de l'abondance spécifique dans les 14 champs en culture de 2004 à 2014.

\begin{tabular}{|c|c|c|c|c|}
\hline Espèces végétales & $\begin{array}{c}\text { Nombre } \\
\text { d'individus } \\
\text { en } 2004\end{array}$ & $\begin{array}{c}\text { Nombre } \\
\text { d'individus } \\
\text { en } 2014\end{array}$ & $\begin{array}{c}\text { Abondance } \\
\text { Relative (\%) }\end{array}$ & $\begin{array}{c}\text { Taux d'évolution } \\
\text { de l'abondance } \\
\text { relative }\end{array}$ \\
\hline Psidium guajava* & 0 & 0 & 0 & $-0,2$ \\
\hline Ficus platyphylla* & 3 & 0 & 0 & $-0,7$ \\
\hline Stereospermum kunthianum* & 1 & 0 & 0 & $-0,2$ \\
\hline Balanites aegyptiaca (Linn.)* & 2 & 0 & 0 & $-0,5$ \\
\hline Faidherbia albida* & 1 & 0 & 0 & $-0,2$ \\
\hline Sterculia setigera* & 2 & 0 & 0 & $-0,5$ \\
\hline Entada africana* & 1 & 0 & 0 & $-0,2$ \\
\hline Ficus ingens* & 2 & 0 & 0 & $-0,5$ \\
\hline Vitex doniana* & 1 & 0 & 0 & $-0,2$ \\
\hline Crataeva religiosa* & 2 & 0 & 0 & $-0,5$ \\
\hline Lannea velutina* & 1 & 0 & 0 & $-0,2$ \\
\hline Anogeissus leiocarpus* & 1 & 0 & 0 & $-0,2$ \\
\hline Strychnos spinosa** & 0 & 1 & 0,3 & 0,3 \\
\hline Erythrina senegalensis $* *$ & 0 & 1 & 0,3 & 0,3 \\
\hline
\end{tabular}




\begin{tabular}{|c|c|c|c|c|}
\hline Crosopterix febrifuga** & 0 & 1 & 0,3 & 0,3 \\
\hline Acacia macrostachya** & 0 & 4 & 1,2 & 1,2 \\
\hline Euphorbia balsamifera** & 0 & 1 & 0,3 & 0,3 \\
\hline Albizia chevalieri $^{* *}$ & 0 & 1 & 0,3 & 0,3 \\
\hline Gardenia erubescens $* *$ & 0 & 1 & 0,3 & 0,3 \\
\hline Maytenus senegalensis $* *$ & 0 & 1 & 0,3 & 0,3 \\
\hline Paveta $\mathrm{sp}^{* *}$ & 0 & 1 & 0,3 & 0,3 \\
\hline Guiera senegalensis** & 0 & 4 & 1,2 & 1,2 \\
\hline Acacia sp ${ }^{* *}$ & 0 & 2 & 0,6 & 0,6 \\
\hline Lannea microcarpa & 36 & 42 & 12,4 & 4,1 \\
\hline Parkia biglobosa & 57 & 40 & 11,8 & $-1,3$ \\
\hline $\begin{array}{c}\text { Acacia nilotica var. } \\
\text { adansonii }\end{array}$ & 8 & 2 & 0,6 & $-1,2$ \\
\hline Mangifera indica & 12 & 5 & 1,5 & $-1,3$ \\
\hline Vitellaria paradoxa & 175 & 127 & 37,5 & $-2,8$ \\
\hline Diospyros mespiliformis & 14 & 9 & 2,7 & $-0,5$ \\
\hline Azadirachta indica & 75 & 38 & 11,2 & $-6,1$ \\
\hline Cassia sieberiana & 0 & 1 & 0,3 & 0,3 \\
\hline Vachellia sieberiana & 0 & 2 & 0,6 & 0,6 \\
\hline Tamarindus indica & 5 & 9 & 2,7 & 1,5 \\
\hline Pterocarpus erinaceus & 3 & 1 & 0,3 & $-0,4$ \\
\hline Bombax costatum & 12 & 5 & 1,5 & $-1,3$ \\
\hline Cassia siamea & 1 & 5 & 1,5 & 1,3 \\
\hline Ficus gnaphalocarpa & 3 & 3 & 0,9 & 0,2 \\
\hline Ziziphus mauritiana & 2 & 3 & 0,9 & 0,4 \\
\hline Calotropis procera & 0 & 1 & 0,3 & 0,3 \\
\hline Sclereocarya birrea & 0 & 2 & 0,6 & $-0,1$ \\
\hline Sterculia setigera & 2 & 4 & 1,2 & 1 \\
\hline Ficus $\mathrm{sp}$ & 0 & 3 & 0,9 & 0,4 \\
\hline Vitex doniana & 1 & 1 & 0,3 & 0,3 \\
\hline Detarium microcarpum & 0 & 3 & 0,9 & 0,4 \\
\hline Lannea acida & 0 & 2 & 0,6 & 0,4 \\
\hline Combretum glutinosum & 1 & 3 & 0,9 & 0,7 \\
\hline Piliostigma thonningii & 2 & 7 & 2,1 & 1,6 \\
\hline Khaya senegalensis & 1 & 2 & 0,6 & 0,4 \\
\hline
\end{tabular}

Source : inventaire floristique et Yaméogo et al., 2005 ; $^{*}$ : espèces disparues ;** : nouvelles espèces. 


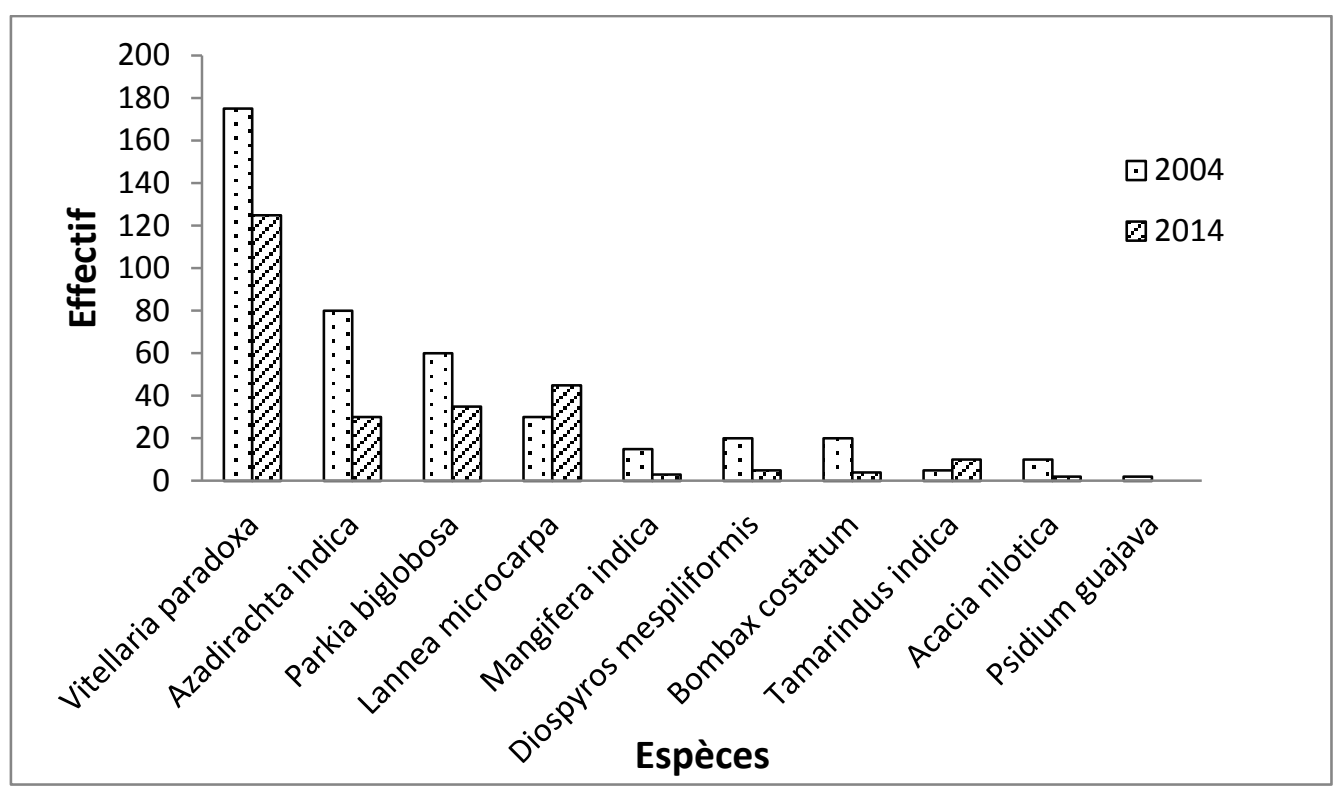

Figure 2: Abondance comparative des espèces végétales entre 2004 et 2014 (source : Yaméogo et al., 2005 et inventaire floristique).

Tableau 3: Moyenne du nombre d'espèces dans 14champs entre 2005 et 2014 et corrélation de Pearson.

\begin{tabular}{|c|c|c|c|c|c|c|c|}
\hline \multicolumn{8}{|c|}{ Paired Samples Statistics } \\
\hline & & Mean & $\mathrm{N}$ & $\begin{array}{c}\text { Std. } \\
\text { Deviation }\end{array}$ & $\begin{array}{c}\text { Std. Error } \\
\text { Mean }\end{array}$ & correlation & Sig \\
\hline \multirow[t]{4}{*}{ Pair 1} & Diversité & 6,5000 & 1 & 2,44163 & ,65255 & 0,192 & 0,511 \\
\hline & spécifique avant & & 4 & & & & \\
\hline & Diversité & 7,2857 & 1 & 4,42769 & 1,18335 & & \\
\hline & spécifique après & & 4 & & & & \\
\hline
\end{tabular}

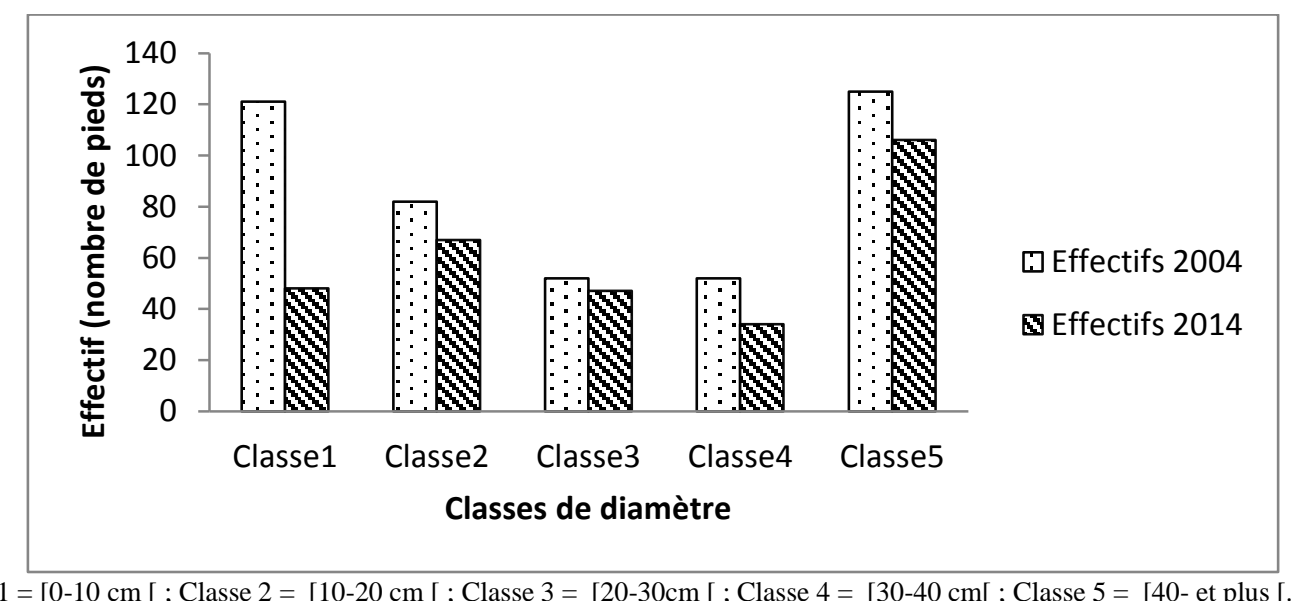

Classe $1=[0-10 \mathrm{~cm}[;$ Classe $2=[10-20 \mathrm{~cm}[;$ Classe $3=[20-30 \mathrm{~cm}[;$ Classe $4=[30-40 \mathrm{~cm}[;$ Classe $5=[40-$ et plus $[$.

Figure 3 : Structure des parcs agroforestiers de Vipalogo de 2004 à 2014 (source : Yaméogo et al., 2005 et inventaire floristique). 


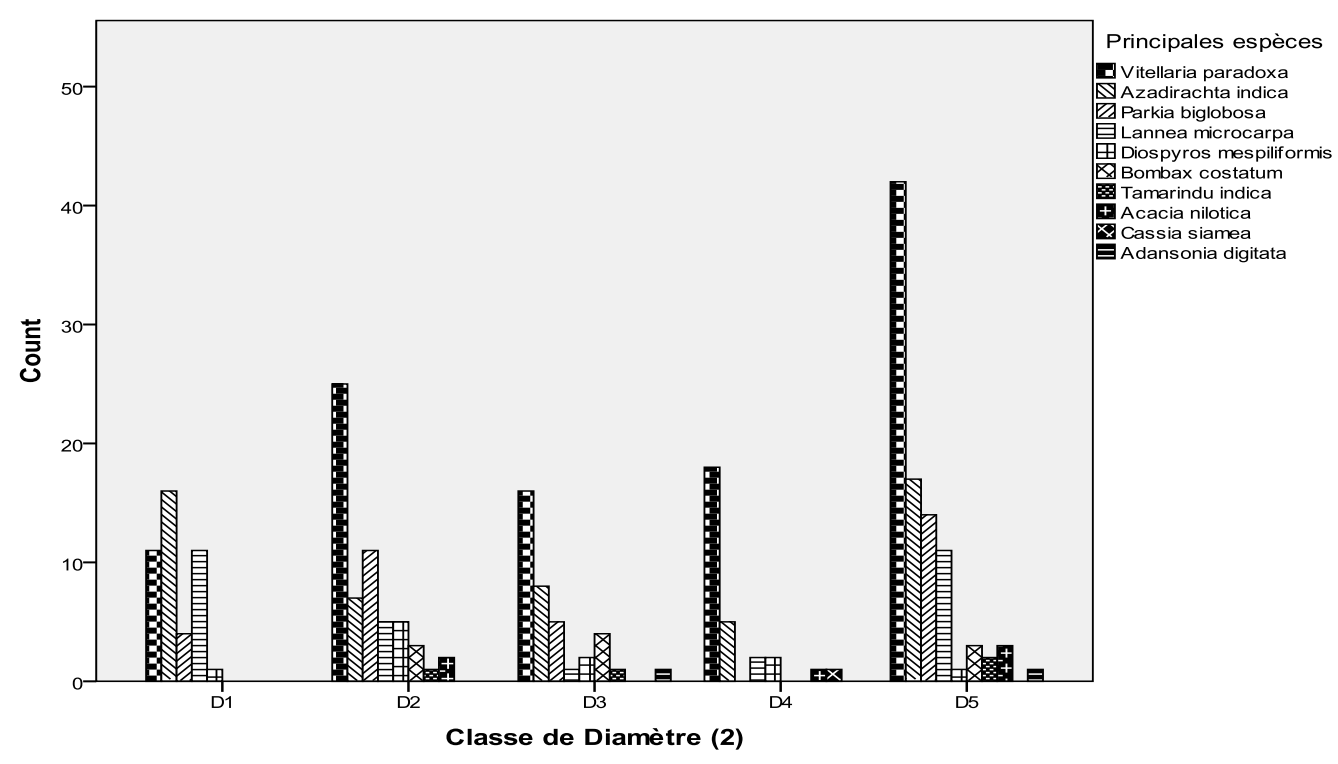

Figure 4 : Effectifs des espèces par classe de diamètre dans les 14 parcs agroforestiers en 2014.

\section{DISCUSSION}

La taille de l'exploitation familiale a faiblement varié depuis 10 ans dans le terroir de Vipalogo. Cette stabilité de la structure familiale est à mettre à l'actif des nombreuses campagnes de sensibilisations conduites sur le contrôle de la natalité ou du slogan «santé mère enfant », par les structures de l'Etat, les ONG et les Organisations de la Société Civile. La précarité de la vie étant liée également à la taille de la famille qui est vue comme une charge, de plus en plus, les populations prennent conscience et adoptent ces nouveaux préceptes sur la gestion familiale. En outre, la proximité de Vipalogo avec la capitale Ouagadougou, permet de nombreux déplacements journaliers des populations vers celle -ci pour la recherche du travail surtout, d'où un accès plus grand à l'information. Cependant si la taille de la famille n'a pas sensiblement augmenté, le nombre de mariages ou de couples formés a augmenté. Cette évolution explique pour 27,5\%, l'extension des superficies des parcs agroforestiers. De plus en plus de nos jours, la possession d'un champ est le signe de l'autonomisation des jeunes couples formés d'où l'augmentation du nombre de champs et des superficies cultivées. Or de par le passé, l'exploitation traditionnelle familiale regroupait plusieurs couples avec des champs communs dont la superficie augmentait en fonction du nombre des membres et la gestion assurée par le père de famille ou l'aîné de la famille (Yameogo, 2008).

Le terroir de Vipalogo, situé sur le plateau central du Burkina Faso, en zone nord soudanienne, est caractérisé par une pluviométrie variant de 700 à $900 \mathrm{~mm}$ par an et des sols ferrugineux tropicaux lessivés (Yaméogo et al., 2005). Les spéculations adaptées à ces caractéristiques pédoclimatiques sont surtout le sorgho, le petit mil, les arachides et le maïs (MEF, 2009). En outre, les spéculations les plus abondamment cultivées demeurent le sorgho et le petit mil qui sont des espèces adaptées à la précarité climatique (Bougma et al., 2018 ; Routier et al., 2011). Il faut noter que malgré la proximité de la ville et l'évolution des mentalités, le mode d'accès à la terre demeure inchangé par rapport à ce qui se faisait en 2004 (Yaméogo et al., 2005). Cependant il faut souligner que les femmes qui deviennent chefs de famille, à la suite du décès de leur mari, conservent le droit sur la terre. 
L'augmentation de la population et la construction de nouveaux logements entrainent une réduction de l'espace agricole à proximité des concessions. Il faut de plus en plus s'éloigner pour avoir la disponibilité en terres cultivables, ce qui explique qu'une forte proportion d'enquêtés possèdent leur parc agroforestier en brousse.

De la présente étude sur la gestion des parcs agroforestiers et leur dynamique évolutive, entre 2004 et 2014, il apparait une évolution de la mentalité paysanne face à certaines réalités liées à la précarité des conditions d'existence. Nos résultats montrent une dégradation des parcs agroforestiers du terroir de Vipalogo de même qu'une évolution des modes de gestion des ressources naturelles.

Les paysans de Vipalogo ont compris qu'il fallait garder plus d'arbres dans les nouvelles défriches (Yaméogo et al., 2005). Mais au fil du temps, ces perceptions paysannes ont évolué. Le champ, en plus de sa contribution à la satisfaction des besoins alimentaires, intervient également dans la satisfaction des besoins des populations en produits ligneux. Dix (10) ans après la mise en culture des quatorze (14) parcs agroforestiers, cette étude montre une autre logique paysanne de gestion des ressources orientée vers la satisfaction des besoins présents, plutôt que vers le souci de durabilité, comme l'avait si bien mentionné Daniel Yves Alexandre (2002). Les arbres des champs sont de plus en coupés pour satisfaire les besoins en bois d'énergie mais surtout ceux en bois de construction. Cette assertion est d'autant plus réelle que l'on note une diminution de $A$. indica dans les parcs agroforestiers. Cette espèce est en effet plantée comme bois de construction (Bationo et al., 2005).

L'agriculture sur brulis est toujours une pratique courante dans la zone. On note une faible mécanisation de l'agriculture (Houmy et al., 2013; Lankoandé 2013; Stéphane, 2013). La coupe du bois qui se faisait prioritairement dans les brousses et secondairement dans les parcs agroforestiers a changé. Elle se fait maintenant directement dans les parcs agroforestiers car la brousse est de plus en plus éloignée du village. Yaméogo et al. (2005) mentionnaient que l'une des contraintes liées à l'approvisionnement en bois de chauffe était l'éloignement des brousses. Dix (10) ans après, cette contrainte de l'éloignement est toujours d'actualité, mais à la seule différence que les intervenants (hommes, femmes et enfants) dans la coupe et l'exploitation des produits forestiers non ligneux, ne s'éloignent plus des habitations pour couper le bois et cueillir les produits forestiers non ligneux (PFNL). L'exploitation du bois et des PFNL se fait beaucoup plus maintenant directement sur les arbres du parc agroforestier. Cela corrobore l'assertion de Karsenty (2008) qui estime que la gestion des espaces forestiers ne peut alors se faire indépendamment de celle des espaces agricoles car tous deux obéissent à la même logique et, doivent participer aux mêmes objectifs de développement durable.

En fait, durant ces dix ans de mise en culture, beaucoup d'arbres ont été complètement coupés et éliminés des champs pour la satisfaction des besoins en bois. Plusieurs ligneux du parc ont été mutilés sévèrement par des éleveurs pasteurs à la recherche de fourrages aériens pour leurs animaux.

Il ressort de l'étude que la durée de dix (10) ans de mise en culture n'a pas influencée significativement la variation du nombre d'espèces dans les quatorze champs, et pourtant, le nombre moyens d'individus d'arbre a diminué significativement dans la même période. Cela s'explique par le fait que généralement la coupe de bois et l'exploitation des PFNL est sélective et ne concerne principalement que certaines espèces. Ce qui fait que les autres espèces sont épargnées.

Les données sur la comparaison des moyennes des effectifs des parcs entre 2004 et 2014, corroborent avec ceux du test $t$ d'échantillons appariés (Tableau 4) qui montrent que la différence entre les effectifs moyens des arbres dans les mêmes parcs agroforestiers était significative et n'est pas due au hasard. Cette différence était l'effet des actions anthropiques notamment le déboisement pour la satisfaction des besoins en bois énergie et l'agriculture itinérante.

De ces résultats on peut conclure que ces parcs agroforestiers n'ont pas connu en réalité de séries évolutives et présentent un aspect de vieux parcs ou parcs agroforestiers matures. 
Tableau 4: Test d'échantillon apparié mesurant le nombre d'espèce dans quatorze champs après dix (10) ans de mise en culture.

\begin{tabular}{|c|c|c|c|c|c|c|c|c|c|}
\hline \multicolumn{10}{|c|}{ Test échantillon apparié } \\
\hline & & \multicolumn{5}{|c|}{ double différence } & \multirow[t]{3}{*}{$\mathrm{t}$} & \multirow[t]{3}{*}{ df } & \multirow{3}{*}{$\begin{array}{l}\text { Sig. (2- } \\
\text { tailed) }\end{array}$} \\
\hline & & \multirow[t]{2}{*}{ Moyenne } & \multirow{2}{*}{$\begin{array}{l}\text { Déviation } \\
\text { standard }\end{array}$} & \multirow{2}{*}{$\begin{array}{l}\text { Erreur } \\
\text { standard } \\
\text { sur la } \\
\text { moyenne }\end{array}$} & \multicolumn{2}{|c|}{ 95\% degré de confiance } & & & \\
\hline & & & & & Min & Max & & & \\
\hline Paire 1 & $\begin{array}{l}\text { Diversité } \\
\text { spécifique } \\
\text { avant - } \\
\text { Diversité } \\
\text { spécifique } \\
\text { après }\end{array}$ &,- 78571 & 4,62732 & 1,23670 & $-3,45745$ & 1,88602 &,- 635 & 13 & ,536 \\
\hline
\end{tabular}

\section{Conclusion}

L'objectif de cette étude était d'analyser l'évolution des modes de gestion des ressources naturelles et la dynamique du potentiel ligneux 10 ans après la création des parcs à Vipalogo. Nos résultats montrent que les parcs agroforestiers de Vipalogo présentent une structure et une composition relativement dégradées depuis leur mise sous culture en 2004. En dix années consécutives sous culture, la structure et le fonctionnement des parcs agroforestiers de Vipalogo ont subi des mutations profondes. On note une baisse de leur diversité spécifique en ligneux. Cette diversité spécifique est passée de 35 espèces en 2004 à 29 espèces en 2014 soit six espèces disparues des parcs. En outre, pendant que les superficies des parcs agroforestiers connaissaient une extension, l'abondance des arbres dans les parcs agroforestiers diminuait, régressant ainsi d'un écart de 131 individus. Le temps de mise sous cultures des parcs agroforestiers de Vipalogo durant dix (10) ans a entrainé une diminution de leur potentiel ligneux. Il apparait alors nécessaire face au recul de la biodiversité dans les parcs du fait de la gestion actuelle des populations que des actions de sensibilisations soient menées pour des activités de boisement et/ou reboisement afin de diminuer la pression sur ces ressources locales à régénération naturelle de plus en plus compromise.

\section{CONFLIT D'INTERETS}

Les auteurs déclarent ne pas avoir de conflit d'intérêts pour ce manuscrit.

\section{CONTRIBUTIONS DES AUTEURS}

GY : Encadrement, orientation de l'étude et correction du manuscrit. HO : Conduite de l'étude sur le terrain, collecte des données, analyses statistiques des données et écriture du manuscrit. BY: Encadrement, orientation de l'étude et correction du manuscrit.

\section{REFERENCES}

Alexandre D-Y. 2002. Initiation à l'Agroforesterie en Zone Sahélienne: les Arbres des Champs du Plateau Central au Burkina Faso. IRD : Karthala. 220 p.

Bationo BA, Yelemou B, Ouedraogo SJ. 2004. Le neem (Azadirachta indica A. Juss.), une espèce exotique adoptée par les paysans du centre- ouest du Burkina Faso. Bois et Forêts Des Tropiques 282(4) : 5-10.

Boffa JM. 2000. Les parcs agroforestiers en Afrique de l'Ouest: clés de la conservation et d'une gestion durable. Unasylva 200, 51. 
Bougma AL, Ouédraogo MN, Sawadogo N, Balma D, Vernoy R. 2018. Perceptions paysannes de l'impact du changement climatique sur le mil dans les zones sahéliennes et soudano-sahéliennes du Burkina Faso. Afrique Sciences, 14 (4): 264-275.

Faure G. 1994. Mécanisation, productivité du travail et risques: le cas du Burkina Faso. Economie Rurale, 219 : 3-11.

Fontes J, Guinko S. 1995. Carte de la végétation et de l'occupation du sol du Burkina Faso. Notice explicative. Ministère de la coopération française, projet Campus, Toulouse, 68 p.

Guegant J, Banoin M, Quesnel A. 2003. Dynamique des populations, disponibilités en terres et adaptation des régimes fonciers : le cas du Niger. Thèse de doctorat, Université Abdou Moumouni de Niamey, Niamey, p. 144.

Grouzis M, Diedhiou, I, Rocheteau A. 1998. Legumes diversity and root symbioses on an aridity grandient in Senegal. Afr. $J$. Ecol., 36: 129-39. DOI: 10.1046/j.13652028.1998.00117.x.

Houmy K, Clarke LK, Ashburner JE, Kienzle J. 2013. Agricultural Mechanization in Sub-saharan Africa: Guidelines for Preparing a Strategy. Integrated Crop Management : Rome.

Sambaré O, Ouedraogo O, Wittig R, Thiombiano A. 2010. Diversité et écologie des groupements ligneux des formations ripicoles du Burkina Faso (Afrique de l'Ouest). Int. J. Biol. Chemi. Sci., 4 (5) : 1782-1800.

Thiombiano A, Kampmann D. 2010. Atlas de la Biodiversité de l'Afrique de l'Ouest Tome II, Burkina Faso. Ouagadougou et Frankfurt/Main. 52p.

Karsenty A. 2008. Des « communautés locales » problématiques. In La Gestion Concertée des Ressources Naturelles. L'Epreuve du Temps, Gret: Karthala, 277-288.

Lankoandé TE. 2013. Analyse de l'influence de la mécanisation agricole sur les possibilités de développement de l'agriculture de conservation dans la région de la boucle du Mouhoun. Mémoire d'ingenieur, Université Nazi Boni, Bobo Dioulasso, p. 59.

Ministère de l'Economie et des Finances. 2009. Recensement Général de la Population et de l'Habitation de 2006 (RGPH-2006), Résultats définitifs, rapport de synthèse des rapports d'analyses, p. 66.

Naboho R. 2007. Analyse des stratégies paysannes dans la gestion des parcs agroforestiers dans la zone céréalière du plateau central et dans la zone cotonnière de la Boucle du Mouhoun. Mémoire de Maîtrise, Université de Ouagadougou, Ouagadougou, p. 92.

Paré S. 2008. Land use dynamics, tree diversity and local perception of dry forest decline in southern Burkina Faso, West Africa. Doctoral thesis, Swedish University of Agricultural Sciences, Umea.

Roudier P, Sultan B, Quirion P, Berg A. 2011. The impact of future climate change on West African agriculture: what does the recent literature say? Global Environmental Change, 21 : 1073-1083. DOI : 10.1016/j.gloenvcha.2011.04.007.

Stéphane SC. 2013. Stratégie de mécanisation de l'agriculture familiale en Afrique subsaharienne. Inclus Etude de cas du Burkina Faso. Mémoire de master IPAD, SupAgro-Montpellier, Montpellier, p.126.

Yaméogo G, Yélémou B, Traoré D. 2005. Pratique et perception paysannes dans la création de parc agroforestier dans le terroir de Vipalogo (Burkina Faso). Biotechnol. Agron. Soc. Environ., 9 (4) : 241-248.

Yaméogo G. 2008. Les Ressources ligneuses et leur gestion dans le terroir de Vipalogo, province du kadiogo, Burkina Faso, étude de Thèse, Université de Cocody, Abidjan, p. 275. 